\title{
Case Report \\ Prenatal and Peripartum Management of Patients with Hypofibrinogenemia Resulted in Two Successful Deliveries
}

\author{
Yuko Teraoka, ${ }^{1}$ Hiroshi Miyoshi, ${ }^{1}$ Kumi Oshima, ${ }^{2}$ Satoshi Urabe, ${ }^{1}$ \\ Norifumi Tanaka, ${ }^{1}$ and Yoshiki Kudo ${ }^{1}$ \\ ${ }^{1}$ Department of Obstetrics and Gynecology, Graduate School of Biomedical Science and Health Sciences, \\ Hiroshima University, Hiroshima, Japan \\ ${ }^{2}$ Department of Hematology and Oncology, Research Institute for Radiation Biology and Medicine, \\ Hiroshima University, Hiroshima, Japan
}

Correspondence should be addressed to Yuko Teraoka; yteraoka@hiroshima-u.ac.jp

Received 26 October 2016; Revised 12 January 2017; Accepted 19 January 2017; Published 14 February 2017

Academic Editor: Dotun Ogunyemi

Copyright (C) 2017 Yuko Teraoka et al. This is an open access article distributed under the Creative Commons Attribution License, which permits unrestricted use, distribution, and reproduction in any medium, provided the original work is properly cited.

\begin{abstract}
Fibrinogen is an essential agent involved in maintaining pregnancy and coagulation. Since inherited fibrinogen disorders introduce greater risks for conditions such as placental abruption and postpartum hemorrhage, careful prenatal and perinatal management is essential for this patient population. We report two cases of successful deliveries in patients with hypofibrinogenemia. Case 1 is of a 26-year-old (gravida 1, para 1) woman. The patient's fibrinogen level increased spontaneously to higher than $300 \mathrm{mg} / \mathrm{dL}$ during pregnancy, without treatment. She delivered at week 38 of gestation, with no complications. Case 2 is of a 30-year-old (gravida 3, para 1) woman. We performed repeated infusions of fibrinogen to maintain the level higher than $100 \mathrm{mg} / \mathrm{dL}$ during pregnancy and at least $200 \mathrm{mg} / \mathrm{dL}$ in the perioperative period; the patient delivered a healthy infant. We identified a new mutation, Hiroshima I $(\gamma 278 \mathrm{Tyr} \rightarrow$ His). It is important to maintain appropriate fibrinogen levels in cases of inherited fibrinogen disorders for successful prenatal and peripartum management.
\end{abstract}

\section{Introduction}

Fibrinogen is a $340 \mathrm{Da}$ glycoprotein synthesized in the liver, which is involved not only in coagulation but also in maintaining pregnancy. Inherited fibrinogen disorders are divided into two different types: quantitative defects, that is, afibrinogenemia or hypofibrinogenemia, and qualitative defects, that is, dysfibrinogenemia. The prevalence of afibrinogenemia is about 1 in 1 million [1]. Mutations related to these disorders have been detected all over the world. The patient with afibrinogenemia is usually identified at birth by the presence of bleeding from the umbilical cord. However, hypofibrinogenemia and dysfibrinogenemia are usually asymptomatic. Appropriate control of the fibrinogen level is necessary for successful prenatal and peripartum management. It is necessary to maintain the fibrinogen level higher than $100 \mathrm{mg} / \mathrm{dL}$ during pregnancy $[2,3]$. After the onset of labor, the fibrinogen level must be maintained to higher than $150 \mathrm{mg} / \mathrm{dL}$ or $200 \mathrm{mg} / \mathrm{dL}$ in case of caesarean section $[2,3]$. Here, we report two cases of hypofibrinogenemia with different course during pregnancy.

\section{Case Report}

Case 1. A 26-year-old (gravida 1, para 1) woman presented with a positive pregnancy test result. She had been diagnosed with hypofibrinogenemia at the age of 23 during blood testing to be a bone-marrow transplantation donor. Her personal and family histories were negative for hemorrhagic or thrombotic episodes. At 25 years of age, the patient delivered a girl at 39 weeks of gestation. Blood tests were performed 
monthly to check the fibrinogen levels. Since her fibrinogen level increased spontaneously during pregnancy, she did not receive infusion therapy. Her prepregnancy fibrinogen level was $76 \mathrm{mg} / \mathrm{dL}$. During the first trimester, it increased gradually to $119 \mathrm{mg} / \mathrm{dL}$ and was more than $300 \mathrm{mg} / \mathrm{dL}$ in the third trimester. She was admitted to our hospital with rupture of the membranes at 38 weeks and 2 days' gestation. Her coagulation tests showed a normal prothrombin time (PT) of $12.2 \mathrm{~s}$ (normal: 10-13 s), activated partial thromboplastin time (APTT) of $28.0 \mathrm{~s}$ (normal: 25-38 s), and fibrinogen concentration of $311 \mathrm{mg} / \mathrm{dL}$ (normal: $200-400 \mathrm{mg} / \mathrm{dL}$ ). After inducing labor with prostaglandin $\mathrm{E}_{2}$, a $3206 \mathrm{~g}$ healthy male infant was delivered. His fibrinogen level was $171 \mathrm{mg} / \mathrm{dL}$. Though the total amount of bleeding was $828 \mathrm{~mL}$ because of atonic bleeding, uterine contraction improved with intravenous oxytocin injection and no complications occurred during puerperium.

Case 2. A 30-year-old (gravida 3, para 1) woman presented with a positive pregnancy test result. She was diagnosed with hypofibrinogenemia at the age of 25 following an emergency caesarean section with her first pregnancy. She was admitted to the previous hospital to induce labor at 40 weeks and 2 days' gestation, and although there were no complications during labor initially, the patient suddenly developed abnormal vaginal bleeding. Although an emergency caesarean section was performed under general anesthesia, because of suspected placental abruption, no surgical and pathological findings were found to confirm this suspicion. The female infant was healthy with a birth weight of $2120 \mathrm{~g}$. Postpartum hemorrhage (PPH) occurred and the fibrinogen level was $60 \mathrm{mg} / \mathrm{dL}$ postoperatively. Immediate transfusion therapy using fresh-frozen plasma (FFP) was administered; this was the only abnormal bleeding episode experienced by the patient. The infant's fibrinogen level was also low, and inherited hypofibrinogenemia was suspected. The patient's history included an artificial abortion at the age of 21 and a spontaneous abortion at 29. She underwent a dilatation and curettage in these two pregnancies at 8 weeks' gestation, with no fibrinogen infusion. She underwent preoperative coagulation tests, only PT and APTT, the first time, and her fibrinogen level was monitored in the perioperative period after she was diagnosed with hypofibrinogenemia the second time. Her fibrinogen level was $66 \mathrm{mg} / \mathrm{dL}$. The surgeries were completed with no complications. The patient's mother received red blood cell (RBC) infusion therapy after delivery; however, there was no other family history of coagulation testing. The patient's two brothers and their families' histories were negative for hemorrhagic and thrombotic episodes. The patient first visited our hospital at 6 weeks' gestation during this pregnancy. Her coagulation tests showed PT of $12.5 \mathrm{~s}$, APTT of $40.1 \mathrm{~s}$, and fibrinogen concentration of $75.9 \mathrm{mg} / \mathrm{dL}$ (normal: $200-400 \mathrm{mg} / \mathrm{dL}$ ). Hematology doctors were consulted, and fibrinogen infusion therapy was started at 7 weeks' gestation. The patient's fibrinogen level was monitored weekly during her pregnancy. She received $3 \mathrm{~g}$ of fibrinogen weekly to maintain a fibrinogen level higher than $100 \mathrm{mg} / \mathrm{dL}[2,3]$. Beginning in the third trimester, physiological elevation of fibrinogen allowed extension of the infusion therapy interval to every three weeks. Fetal growth slowed at 27 weeks of gestation, and a diagnosis of fetal growth restriction (FGR) was made. The patient was hospitalized at 31 weeks' gestation to monitor FGR, because the fetal growth worsened: the estimated fetal weight (EFW) below -2.5 Standard Deviation (SD) from the population standard. However, the fetus grew at the same rate $(-2.5 \mathrm{SD})$ as the fetal growth curve during hospitalization. Doppler ultrasound of fetal blood vessels was normal. A selective caesarean section was performed at 36 weeks and 2 days' gestation under general anesthesia to avoid the risk of bleeding on the recommendation of the anesthesiologist [4]. The male infant had Apgar scores of 8 and 10 at 1 and $5 \mathrm{~min}$, respectively, with a birth weight of $1724 \mathrm{~g}$. The patient received $3 \mathrm{~g}$ of fibrinogen for $3 \mathrm{con}-$ secutive days, 2 days before the surgery, to maintain the fibrinogen level higher than $200 \mathrm{mg} / \mathrm{dL}$ in the perioperative period. The fibrinogen level was $228 \mathrm{mg} / \mathrm{dL}$ on the day of operation. Total bleeding during the operation was $260 \mathrm{~mL}$. The placenta was hypoplastic, with a weight of $350 \mathrm{~g}$ and marginal insertion of the cord. Since fibrinogen level of the infant persisted at $40-50 \mathrm{mg} / \mathrm{dL}$, he was also suspected to have inherited hypofibrinogenemia, as was the case with his sister. The postoperative course was uneventful. One month after delivery, the patient's fibrinogen level decreased to $68 \mathrm{mg} / \mathrm{dL}$.

We were unable to get patient consent in case 1; therefore, genetic analysis was only conducted in case 2 . Her functional fibrinogen level was $112.0 \mathrm{mg} / \mathrm{dL}$ and immunological level was $115.7 \mathrm{mg} / \mathrm{dL}$; therefore, congenital hypofibrinogenemia was suspected. A new mutation ( $\gamma 278 \mathrm{Tyr} \rightarrow$ His $)$ was identified as leading to hypofibrinogenemia. Inherited fibrinogen disorders are named after the city where the patient lives or after the city in which the patient was evaluated. Therefore, this case was named "Hiroshima I." The same mutation was also detected in her two children.

\section{Discussion}

Inherited fibrinogen disorders are very rare; the number of certified patients was 70 according to the national survey on coagulation disorders 2015 [4]. Inherited fibrinogen disorders are divided into two different types: quantitative defects (i.e., afibrinogenemia or hypofibrinogenemia) and qualitative defects (i.e., dysfibrinogenemia) (Table 1). No complications were observed during the pregnancy in case 1 , whereas abnormal bleeding occurred after the caesarean section in case 2. Coagulation tests for congenital hypofibrinogenemia and dysfibrinogenemia are usually normal, with the exception of fibrinogen levels [1]. Two measurement methods (PT-derived method and immunoturbidimetry) are used to distinguish these two conditions. The PT-derived method can detect functional fibrinogen level and immunoturbidimetry can reveal immunologic fibrinogen level $[1,5]$. Hypofibrinogenemia shows a low level of fibrinogen in both methods and dysfibrinogenemia only demonstrates low functional assay. The functional and immunological level in case 2 showed a 
TABLE 1: Characteristics of inherited fibrinogen disorders.

\begin{tabular}{|c|c|c|c|}
\hline & Afibrinogenemia & Hypofibrinogenemia & Dysfibrinogenemia \\
\hline Transmission & $\mathrm{AR}$ & $\mathrm{AD} / \mathrm{AR}$ & $\mathrm{AD}$ \\
\hline Fibrinogen level (mg/dL) & $<10$ & $70-100$ & Lower limit of normal \\
\hline \multirow{2}{*}{ Laboratory } & PT-APTT-TT & PT: normal or prolonged & \multirow{2}{*}{ PT-APTT: normal } \\
\hline & Markedly prolonged & APTT: normal & \\
\hline \multirow{6}{*}{ Symptoms } & Umbilical cord bleeding & \multirow{6}{*}{$\begin{array}{c}\text { No symptoms } \\
\text { Abnormal bleeding } \\
\text { after trauma or operation }\end{array}$} & \multirow{6}{*}{$\begin{array}{c}\text { No symptoms } \\
\text { Abnormal bleeding } \\
\text { after trauma or operation } \\
\text { Thrombosis }\end{array}$} \\
\hline & Recurrent abortion & & \\
\hline & Cutaneous bleeding & & \\
\hline & Intracranial bleeding & & \\
\hline & Gastrointestinal bleeding & & \\
\hline & Hypermenorrhea & & \\
\hline
\end{tabular}

AR; Autosomal recessive, AD; Autosomal dominant, PT; prothrombin time, APTT; actinated partial thromboplastin time, TT; thrombin time.

proportional decrease; therefore, congenital hypofibrinogenemia was suspected.

Fibrinogen also plays an important role in adhesion between the placenta and the uterus; therefore, while it is not essential for implantation, it is necessary for maintaining pregnancy. According to some case reports, pregnancy in the patients with afibrinogenemia will result in abortion without fibrinogen infusion therapy by at least 5 weeks' gestation $[2,3]$. Moreover, fibrinogen disorders can cause recurrent abortion, subchorionic hematoma, placental abruption, and PPH $[2,3]$. It is known that the minimal fibrinogen level for maintaining pregnancy is above $60 \mathrm{mg} / \mathrm{dL}[2,3]$. As fibrinogen decreases under threatened premature labor or inflammation, it is necessary to maintain the fibrinogen level higher than $100 \mathrm{mg} / \mathrm{dL}$ during pregnancy $[2,3]$. After the onset of labor, the fibrinogen level must be maintained to higher than $150 \mathrm{mg} / \mathrm{dL}$ or $200 \mathrm{mg} / \mathrm{dL}$ in case of caesarean section $[2,3]$. The fibrinogen level increased spontaneously to higher than $200 \mathrm{mg} / \mathrm{dL}$ during pregnancy in case 1 ; therefore, she did not receive infusion therapy. In case 2 , the patient received $3 \mathrm{~g}$ of fibrinogen (half-life of 3-4 days) once a week through the second trimester (Figure 1, Table 2). Beginning in the third trimester, the physiological elevation of fibrinogen allowed extension of the infusion therapy interval to every 3 weeks (Figure 1). The fibrinogen level after surgery stayed higher than $150 \mathrm{mg} / \mathrm{dL}$. Fetal growth restriction occurred, the same as with her previous one. Pathology of the placenta revealed no specific information leading to FGR, such as thrombus or infarction. We did not find out the literature showing relationship between FGR and hypofibrinogenemia and fibrinogen infusion therapy. Marginal insertion of the cord might be one of the reasons for FGR in this case.

Fibrinogen consists of three polypeptide chains (A2 $\alpha$, $\mathrm{B} \beta$, and $\gamma$ ) encoded by three genes (FGA, FGB, and FGG, resp.) located on the long arm of chromosome 4 [6]. Afibrinogenemia is caused by variations in the FGA, FGB, and FGG genes [7]. Afibrinogenemia is associated with homozygous or compound heterozygous mutation. On the other hand, hypofibrinogenemia is usually related to heterozygous

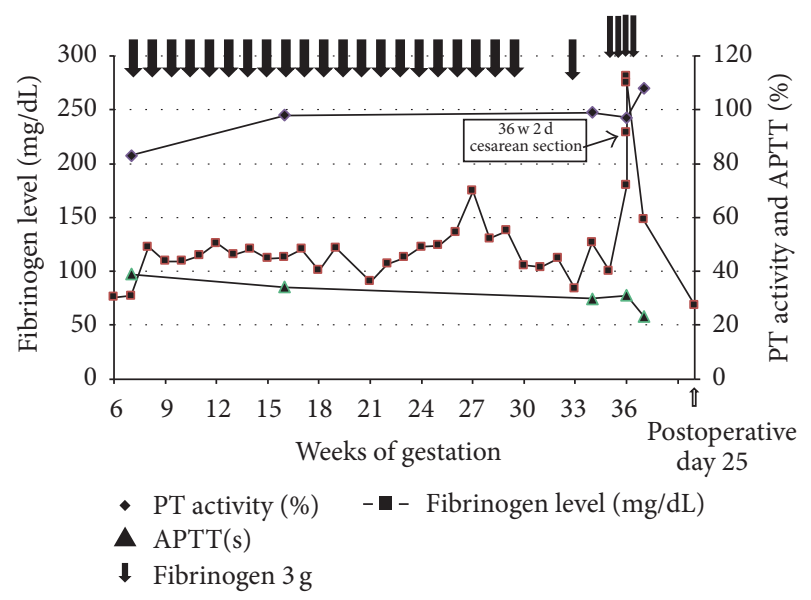

FIgURE 1: Perinatal course and fibrinogen infusion therapy in case 2.

mutation. Mutations causing afibrinogenemia and hypofibrinogenemia in FGA gene are mainly deletions, frameshift, nonsense, or splicing mutations [7]. In contrast, mutations in FBG and FGG include an excess of missense mutation [7]. Genetic analyses have shown the mechanisms regulating the production of fibrinogen. For example, the Matsumoto IV ( $\gamma$ 153 Cys $\rightarrow$ Arg) mutation affects the proximal A region of the $\gamma \mathrm{D}$ domain resulting in abnormal folding without intrachain disulphide bond $[4,8]$. In case 2 , genetic analysis revealed a new mutation ( $\gamma 278 \mathrm{Tyr} \rightarrow$ His) named as Hiroshima I on exon 8 in FGG which can affect mRNA splicing or stability or protein synthesis, assembly, and secretion.

We reported two cases of hypofibrinogenemia with different courses during pregnancy. The personal and family history must be checked carefully in case of fibrinogen disorders, as occasionally hereditary abnormalities are found, such as in case 2. For the patients with the fibrinogen disorders, the fibrinogen level must be measured continuously and infusion therapy is essential not only maintaining the pregnancy but also preventing $\mathrm{PPH}$ and other complications. 
TABLE 2: Fibrinogen level during pregnancy in case 2.

\begin{tabular}{lc}
\hline Weeks of gestation & Fibrinogen level (mg/dL) \\
\hline 9 & 109.2 \\
10 & 109.2 \\
11 & 114.6 \\
12 & 125.9 \\
13 & 115.5 \\
14 & 120.7 \\
15 & 112 \\
16 & 112.9 \\
17 & 120.7 \\
18 & 100.6 \\
19 & 121.8 \\
21 & 90.5 \\
22 & 106.8 \\
23 & 112.9 \\
24 & 122.8 \\
25 & 123.9 \\
26 & 136.5 \\
27 & 174.4 \\
28 & 130.5 \\
29 & 137.8 \\
30 & 105.5 \\
31 & 103.7 \\
32 & 112 \\
33 & 83.6 \\
34 & 126.5 \\
POD25 & 100.2 \\
Operation day & 180.3 \\
POD4 & 228.8 \\
POD7 & 281.4 \\
35 & 274.7 \\
& 147.8 \\
& 68 \\
\hline &
\end{tabular}

POD: postoperative day.

\section{Competing Interests}

The authors declare that there is no conflict of interests regarding the publication of this paper.

\section{Acknowledgments}

The authors wish to thank Dr. Okumura at the Department of Biomedical Laboratory Sciences, School of Health Sciences, Shinshu University, for genetic analysis.

\section{References}

[1] S. S. Acharya and D. M. Dimichele, "Rare inherited disorders of fibrinogen," Haemophilia, vol. 14, no. 6, pp. 1151-1158, 2008.

[2] T. Kobayashi, T. Terao, and J. Takamatsu, "Congenital afibrinogenemia: prenatal and peripartum management," Japanese Journal of Thrombosis and Hemostasis, vol. 12, pp. 57-65, 2001.
[3] K. Takata, T. Asahina, T. Kobayashi et al., "A case report of congenital afibrinogenemia with successful prenatal and peripartum management," The Japanese Journal of Obstetrical, Gynecological \& Neonatal Hematology, vol. 15, pp. 53-58, 2006.

[4] K. Watanabe, Y. Nakajima, and M. Sakanashi, "Perioperative management of a patient with congenital hypofibrinogenemia who underwent myomectomy and cesarean section," The Journal of Japan Society for Clinical Anesthesia, vol. 33, no. 2, pp. 242246, 2013.

[5] N. Okumura and F. Terasawa, "Analyses for dysfunctional fibrinogenemias and hypo/afibrinogenemias; abnormality of function and synthesis of fibrinogen," The Shinshu Medical Journal, vol. 57, pp. 145-154, 2009.

[6] G. J. Maghzal, S. O. Brennan, V. M. Homer, and P. M. George, "The molecular mechanisms of congenital hypofibrinogenaemia," Cellular and Molecular Life Sciences, vol. 61, no. 12, pp. 1427-1438, 2004.

[7] A. D. Mumford, S. Ackroyd, R. Alikhan et al., "Guideline for the diagnosis and management of the rare coagulation disorders: A United Kingdom haemophilia centre doctors' organization guideline on behalf of the British committee for standards in haematology," British Journal of Haematology, vol. 167, no. 3, pp. 304-326, 2014.

[8] F. Terasawa, N. Okumura, K. Kitano et al., "Hypofibrinogenemia associated with a heterozygous missense mutation $\gamma 153$ Cys to Arg (Matsumoto IV): in vitro expression demonstrates defective secretion of the variant fibrinogen," Blood, vol. 94, no. 12, pp. 4122-4131, 1999. 


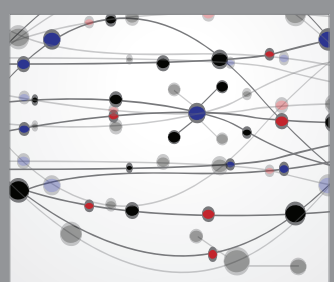

The Scientific World Journal
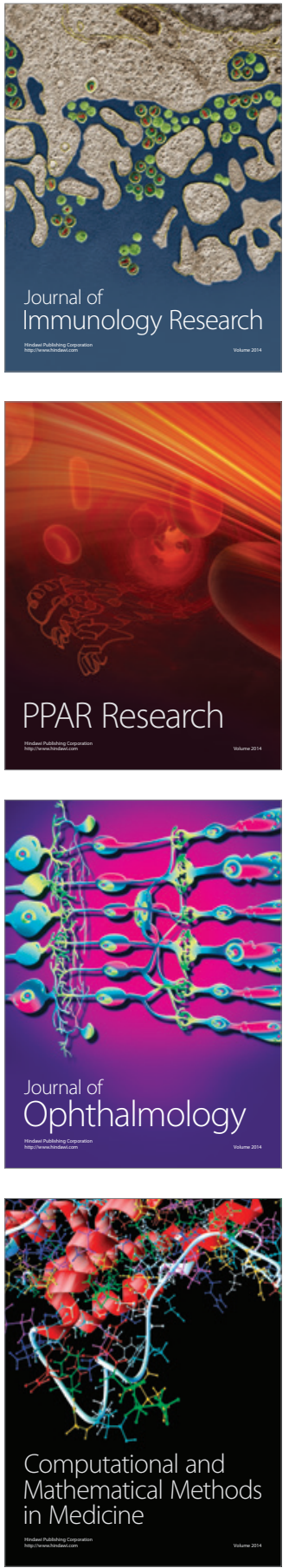

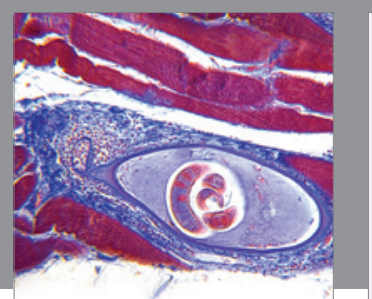

Gastroenterology Research and Practice
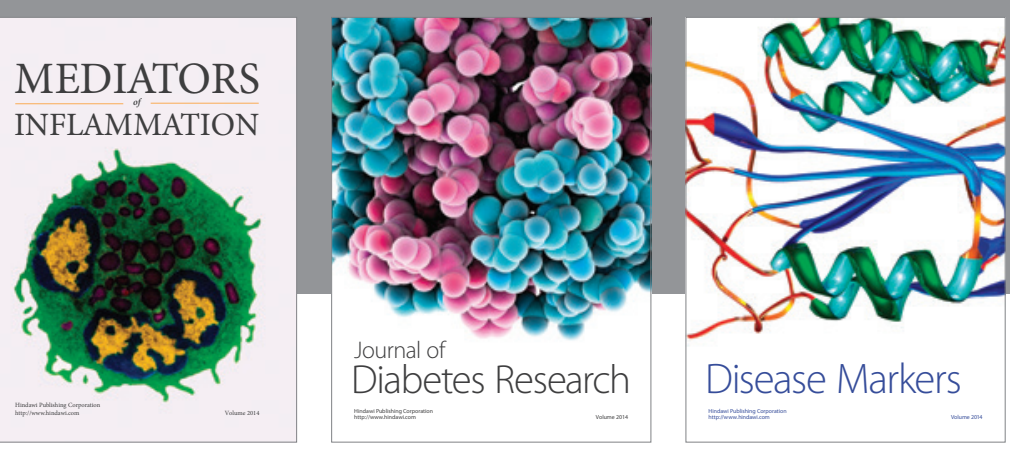

Disease Markers

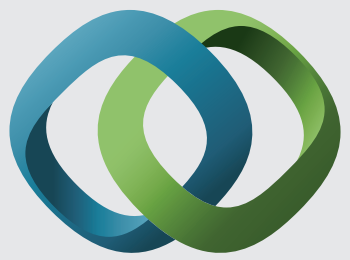

\section{Hindawi}

Submit your manuscripts at

https://www.hindawi.com
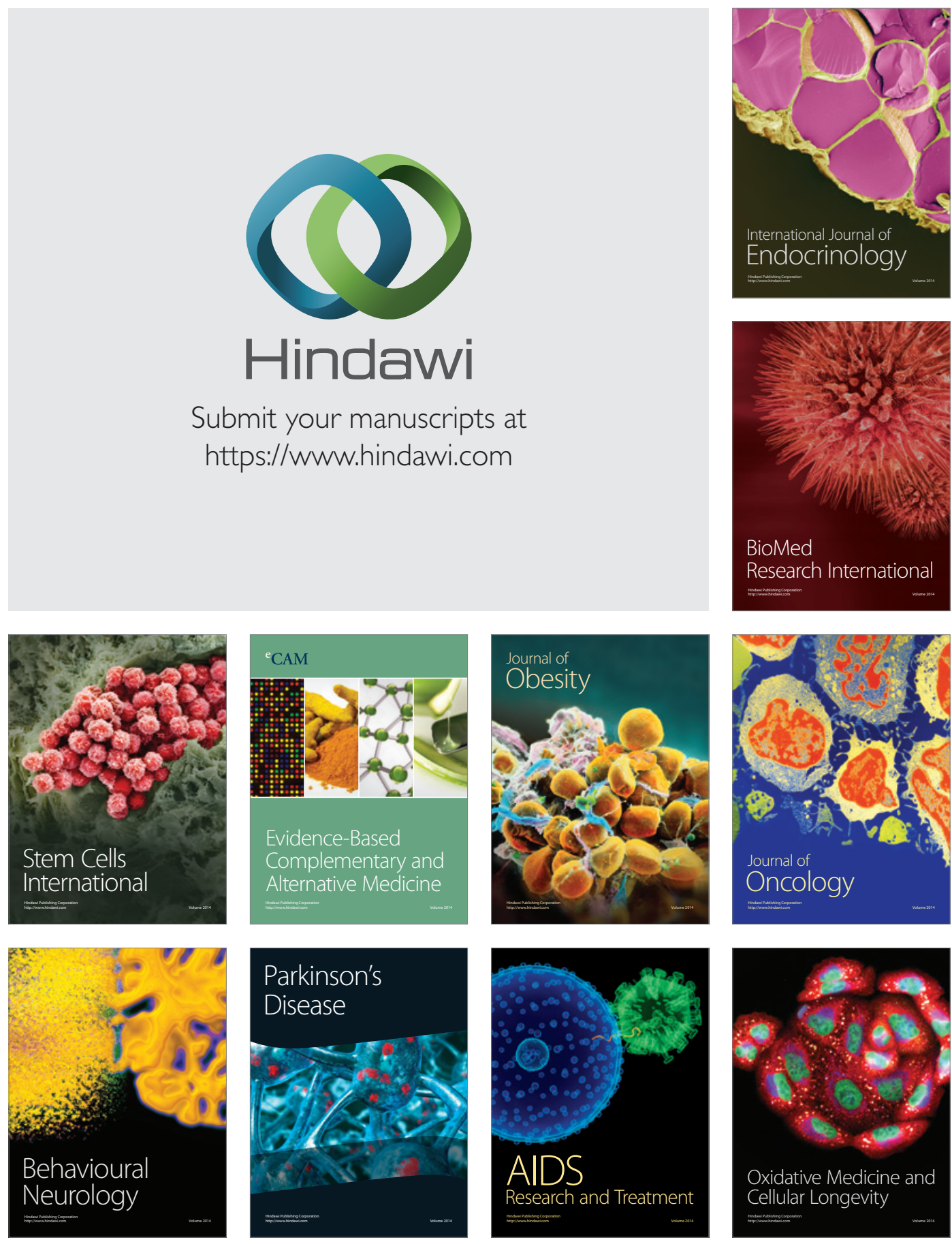